\title{
Quantitative Bestimmung der Phosphorfleischsäure.
}

\section{Von}

Balke und Ide.

(Aus dem chemischen Laboratorium des physiologischen Instituts der Universität Leipzig.) (Der Redaction zugegangen am 9. Dccember 1895.)

Um die physiologischen Verhältnisse der Phosphorfleischsäure näher studieren zu können, war es vor Allem erforderlich, eine Methode zur quantitativen Bestimmung der Phosphorfleischsäure aufzusuchen, eine Arbeit, die uns Herr Dr. Siegfried in dankenswerther Weise überliess. Wir geben im Folgenden den Gang wieder, den wir bei Ausarbeitung dieser quantitativen Bestimmungsmethode eingeschlagen haben.

Versuch I. Zur Bestimmung der Phosphorfleischsäure benutzten wir die Eisenverbindung derselben, das Carniferrin "). Dasselbe stellt man gewöhnlich dar, indem man den von Phosphaten befreiten Fìeischextract mit Eisenchlorid versetzt und auf kocht; da jedoch ein Ueberschuss von Eisenchlorid das bereits gebildete Carniferrin wieder auflöst, so benutzten wir zuerst zur Fällung des Carniferrins anstatt Eisenchlorid Ferriammoniakalaun. Ein Ueberschuss dieses Eisensalzes löst bereits gefälltes Carniferrin nicht wieder auf. Nun erhält man aber schon beim Kochen des Alaunes in Wasser allein einen Niederschlag, der zwar nicht untersucht wurde, jedoch wahrscheinlich N-haltig ist, da, wie aus späteren Versuchen hervorgeht, die erhaltenen Resultate zu hoch sind. Wir verfuhren bei diesem Versuche in der Weise, dass die Extractlösung mit

1) Arch. f. Anat. u. Physiol., Physiolog. Abthlg., 1894, S. 401. 
frisch gefälltem Baryumcarbonat und Baryumchlorid längere Zeit (ca. 4 Stunden) auf dem Wasserbade erhitzt und das Filtrat von den Phosphaten, welches sauer reagirte, mittelst Natriumsulfat vom Baryt befreit wurde. Das baryumfreie Filtrat wurde nun kochend mit Ferriammoniakalaun versetzt bis zum Eintreten einer deutlichen Ferrireaction, die durch Rhodankalium festgestellt wurde. Der erhaltene Carniferrin-Niederschlag wurde gewaschen, bei $110^{\circ}$ getrocknet und sein $\mathrm{N}$-Gehalt nach der Kjeldahl'schen Methode bestimmt. Die Menge des Carniferrins, beziehungsweise des darin enthaltenen Stickstoffs wurde bei allen Versuchen immer auf Fleischsäure, $\mathrm{G}_{10} \mathrm{H}_{15} \mathrm{~N}_{3} \mathrm{O}_{5}$, berechnet, deren Stickstoffgehalt $16,33 \%$ beträgt. Es ergaben:

\begin{tabular}{l||c|c|c|c|c}
\hline \hline Fleischextract. & $\begin{array}{c}\text { Carniferrin } \\
\text { in gr. }\end{array}$ & $\begin{array}{c}\text { Carniferrin } \\
\text { in \%. }\end{array}$ & $\begin{array}{c}\text { stickstoff } \\
\text { im Carniferrin. }\end{array}$ & Fleischsāure. \\
\hline a) & $0,9612 \mathrm{gr}$. & $0,3131 \mathrm{gr}$. & $32,6 \%$ & - & - \\
b) & $0,4806 \%$ & 0,1638 & $34,08 \%$ & $3,75 \%$ & $16,5 \%$
\end{tabular}

Da, wie aus weiter unten folgenden Versuchen ersichtlich, der Fleischsäure-Gehalt in Liebig's Fleischextract durchschnittlich $6,10 \%$ beträgt, so zeigt sich, dass man bei Anwendung von Ferriammoniakalaun $\mathrm{zu}$ viel Stickstoff, bezw. Fleischsäure findet und Ferriammoniakalaun daher zur quantitativen Bestimmung der Phosphorfleischsäure nicht brauchbar ist.

Versuch II. Bei diesem Versuch wurde die Ausfällung des Carniferrins mit Eisenchlorid bewirkt und zwar wurde ein Ueberschuss von Eisenchlorid durch die Tüpfelprobe mit Rhodankalium zu vermeiden gesucht. Die Extractlösung wurde in der Kälte genau mit Natronlauge neutralisirt, bezw. sehr. leicht alkalisch gemacht und die Phosphate mit Baryumchlorid ausgefällt. Alsdann wurde $\mathrm{zu}$ dem kochenden Filtrat eine Lösung von Eisenchlorid (10 gr. festes, gewöhnliches Eisenchlorid in 1000 gr. Wasser) gegeben, einige Sekunden lang kochen gelassen und dann die Tüpfelprobe mit Rhodankalium gemacht. 
Es zeigte sich, dass eine halbe Minute Kochen genügt, um das Carniferrin zu bilden, bezw. eine Ferrireaction wieder verschwinden zu lassen. Die Niederschläge wurden alsdann abgesaugt, chlorfrei gewaschen, bei $105^{\circ}$ getrocknet und der $\mathrm{N}$-Gehalt nach Kjeldahl bestimmt. Es ergaben sich hierbei folgende Resultate:

\begin{tabular}{|c|c|c|c|c|c|c|}
\hline & $\begin{array}{l}\text { Fleisch- } \\
\text { extract. }\end{array}$ & $\begin{array}{l}\text { Carniferrin } \\
\text { in gr. }\end{array}$ & $\begin{array}{l}\text { Carniferrin } \\
\text { in } \% .\end{array}$ & $\begin{array}{l}\text { Stickstoff } \\
\text { im Carnif. }\end{array}$ & Fleischs. & $\begin{array}{l}\text { Eisen- } \\
\text { chlorid. }\end{array}$ \\
\hline a) & $0,9304^{\mu} \mathrm{gr}$. & $0,1289 \mathrm{gr}$. & $13,9 \%$ & $6,19 \%$ & $5,26 \%$ & 39 cbrm. \\
\hline b) & 0,9304 & 0,1439 & $15,5 \gg$ & - & - & $35 \gg$ \\
\hline c) & $0,9304 \gg$ & $0,1563 \gg$ & $16,8 》$ & $6,10 \%$ & $6,27 \%$ & 33 \\
\hline d) & $0,9304 \gg$ & 0,1526 & $16,4 »$ & $5,90 \gg$ & $5,92 »$ & 32 \\
\hline
\end{tabular}

Aus der letzten Spalte ist ersichtlich, dass zur Fällung immer weniger und weniger Eisenchlorid genommen wurde; in den beiden letzten Fällen c) und d) war die Reaction mit Rhodankalium nicht sehr deutlich; in grösseren Mengen des abgesaugten Filtrats jedoch gab hinzugefügtes Rhodankalium noch eine deutliche Röthung. Sehr bemerkenswerth ist die Thatsache, dass gerade die Proben, zu welchen am wenigsten Eisenchlorid gesetzt war, die höchstprocentigen Niederschläge lieferten. Sobald man einen grösseren Ueberschuss von Eisenchlorid zufügt, wird das Filtrat wieder stark röthlich; man kann daher die Helligkeit, bezw. Klarheit des Filtrates neben der Ferrireaction als zweites Zeichen der völligen Ausfällung des Carniferrins annehmen. Bei einer bestimmten Grenze der zugesetzten Eisenchloridmenge geht das Carniferrin beim Kochen sehr rasch wieder in Lösung, wobei, wie schon erwähnt, das Filtrat roth wird; für $0,9304 \mathrm{gr}$. Extract lag diese Grenze bei ungefähr $40 \mathrm{cbcm}$.: $0,9304 \mathrm{gr}$. Extract, mit ca. $40 \mathrm{cbcm}$. Ejsenchlorid versetzt, gab ein schon ziemlich gefärbtes Filtrat und lieferte nur 8,3\% Niederschlag anstatt $15 \%$. Endlich suchten wir noch auf verschiedene Weisen zu zeigen, dass in dem klaren Filtrat vom Carniferrin nicht noch erhebliche Mengen Carniferrin gelöst bleiben. Diese Proben wurden mit dem Filtrat vom Niederschlag b) ausgeführt. Dieses Filtrat gab mit Rhodankalium eine geringe Ferrireaction; Eisenchlorid 
war also genügend zugesetzt; es konnte jedoch noch Carniferrin in Lösung sein, da das Filtrat stark sauer reagirte. Es wurde daher mit Baryumcarbonat gefällt, wobei natürlich alles Eisenchlorid zugleich mit eventuell vorhandenem aufgelösten Carniferrin ausfiel. Der Niederschlag wurde abfiltrirt und gewaschen. Eine Probe desselben, qualitativ auf Stickstoff untersucht, erwies sich als stickstofffrei.

Versuch III. Bei diesem Versuch wurde die Extractlösung nach der Behandlung mit Baryumchlorid schwach sauer gelassen, anstatt schwach alkalisch gemacht zu werden. Alsdann wurden zum kalten Filtrat vom Phosphatniederschlag $30 \mathrm{cbcm}$. der 1 procentigen Eisenchloridlösung hinzugefügt, da sich ja bei dem vorigen Versuch ergeben hatte, dass diese Menge Eisenchlorid zur Fällung des Carniferrins von 0,9304 gr Extract genügt. In der Kälte entsteht gar kein Niederschlag, sondern erst beim Kochen, indem das Eisenchlorid jedenfalls zuerst oxydirend und zwar beim Erhitzen wirkt. Es ergaben hierbei 0,9304 gr. Fleischextract 0,1656 gr. $=17,8 \%$ Carniferrin, dessen $\mathrm{N}$-Gehalt $5,6 \%$ betrug, was einem Fleischsäuregehalt des Fleischextracts von 6,1\% gleichkommt. Es entspricht dieser Werth auf das Beste den Resultaten des dritten Versuchs. Das Filtrat war hell und klar und gab keinen weiteren Niederschlag weder beim Kochen noch beim $\mathrm{Ab}$ stumpfen der saueren Reaction. Das Filtrat zeigte eine schwache Ferrireaction.

Versuch IV. Die Bestimmungen der Phosphorfleischsäure wurden hier nicht in käuflichen Fleischextract, sondern in selbstbereiteten Lösungen der Extractivstoffe von thierischen Organen gemacht. Es wurden zu diesem Zwecke immer ca. $11 \%$ Kilo des betreffenden Organes mittelst einer Maschine zerkleinert, dann genau 1 Kilo abgewogen und zuerst zweimal mit je 2 Liter, dann einmal mit einem Liter Wasser auf dem Wasserbade bei $50^{\circ}$ bis $60^{\circ}$ unter gutem Umrühren ungefähr eine Stunde lang digerirt und durch ein Colirtuch gepresst. Die vereinigten Filtrate wurden dann behufs Coagulation der Eiweisskörper aufgekocht und nach dem Erkalten nochmals filtrirt. Aus dem Filtrate wurden die Phosphate durch eine 
Lösung von Calciumchlorid und Ammoniak ausgefällt. Das neutral gemachte Filtrat wurde nunmehr zum Kochen erhitzt und mit einer 1 procentigen Lösung von Eisenchlorid versetzt, das man am besten aus einer Bürette hinzulässt. Durch fortwährende Tüpfelproben mit Rhodankalium sucht man einen grossen Ueberschuss von Eisenchlorid zu vermeiden, lässt, wenn Ferrireaction eintritt, ein paar Minuten kochen und sieht dann, ob die Ferrireaction bestehen bleibt. Zuerst wird dieselbe nach dem Kochen immer wieder verschwunden sein, da sich ja, wie wir aus früheren Versuchen gesehen haben, das Carniferrin erst bein Kochen bildet. Bleibt jedoch nach dem Kochen die Ferrireaction noch vorhanden, so hat man genügend Eisenchlorid zugefügt. Das Filtrat ist dann hell und klar. Bei einigen Bestimmungen wurde absichtlich ein grösserer Ueberschuss von Eisenchlorid zugefügt; alsdann geht Carniferrin in Lösung und das Filtrat wird roth. (Vergl. Versuch II.) Setzt man jedoch nach dem Kochen etwas Ammoniak zu, so dass die Lösung aber immer noch schwach sauer reagirt, so wird das Filtrat wieder klar, indem das überschüssige Eisenchlorid als Eisenhydroxyd ausgefällt wird. Die Ferrireaction verschwindet hierbei allmälig. Man muss jedoch einen Ueberschuss von Ammoniak vermeiden, da durch dasselbe das Carniferrin zersetzt wird. Da durch den Zusatz von Ammoniak neben dem gelösten Carniferrin auch noch Eisenhydroxyd ausfällt, so erhält man einen grösseren Eisenniederschlag, der aber entsprechend weniger $\mathrm{N}$-reich ist. Die Flüssigkeit mit dem Eisenniederschlag wurde dann aus den Schalen in grössere Gefässe gegossen, wo sich der Niederschlag sehr leicht absetzte. Der Niederschlag wurde nun durch Dekantiren ausgewaschen, bis er nicht mehr die Salzsäurereaction gab. Bei dem Auswaschen kann es nun geschehen, dass das zuvor klare Waschwasser allmälig eine starke Opalescenz zeigt, die wahrscheinlich von kolloidgelöstem Eisenhydroxyd herrührt und zwar ist dies das von Péan St. Gilles ${ }^{1}$ ) beschriebene. sogenannte lösliche Eisenhydroxyd, welches gewöhnlich durch

1) A. ch. [3] 46, 47; J. 1855, 401. Nach Dammer, Handbuch delanorgan. Chemie. 
längeres Kochen des durch Ammoniak aus Eisenchlorid entstandenen Eisenhydroxyds dargestellt wird. Dieses Eisenhydroxyd ist in Säuren enthaltendem Wasser unlöslich, löslich jedoch in reinem Wasser. Wenn nun, wie oben angegeben, das überschüssige Eisenchlorid durch Ammoniak kochend ausgefällt worden ist, so ist die Bildung von löslichem Eisenhydroxyd leicht erklärlich und man sieht auch ein, wesshalb die Lösung zuerst klar, nach längerem Auswaschen aber opalisirend wird. Wenn man jedoch genügend lang auswäscht, so verschwindet die Opaleszenz wieder. Die durch Dekantiren ausgewaschenen Eisenniederschläge wurden am Ende noch centrifugirt, mit Alkohol und Aether behandelt, bei $105^{\circ}$ getrocknet und der $\mathrm{N}$-Gehalt derselben nach $\mathrm{Kj}$ eldahl bestimmt. Auf diese Weise wurden folgende Resultate erhalten:

\begin{tabular}{|c|c|c|c|c|c|}
\hline Organ. & Gewicht. & $\begin{array}{l}\text { Carniferrin } \\
\text { in gr. }\end{array}$ & $\begin{array}{c}\text { Carniferrin } \\
\text { in } \% .\end{array}$ & $\begin{array}{l}\text { Stickstoff } \\
\text { in Oarnif. }\end{array}$ & $\begin{array}{l}\text { Fleisch- } \\
\text { säure. }\end{array}$ \\
\hline a) Herz. & $1000 \mathrm{gr}$. & $4,2158 \mathrm{gr}$. & $0,422 \%$ & $4,50 \%$ & $0,116 \%$ \\
\hline$=$ b) Herz. & 1000 & $4,55577 \gg$ & $0,456 \gg$ & 3,77 & 0,105 》 \\
\hline o c) Leber. & $1000 »$ & $0,4566 \gg$ & $0,045 \gg$ & $5,45 》$ & $0,015 \gg$ \\
\hline$\Xi$ d) Leber & 1000 & $0,4304 \gg$ & $0,043 \gg$ & $5,50 \gg$ & $0,015 \gtrsim$ \\
\hline (e) Niere . & 1000 & $1,4047 \gg$ & 0,140 , & $5,06 \gg$ & $0,043 »$ \\
\hline f) Niere & $73 »$ & $0,5642 \gtrsim$ & 0,773 » & $4,32 》$ & 0,205 » \\
\hline$\Xi\{\mathrm{g})$ Herz. & $133 \gg$ & $0,9282 \gg$ & 0,690 & $5,92 »$ & $0,253 »$ \\
\hline (h) Leber & $439 \gg$ & 2,1574 & $0,500 »$ & $6,03 \bowtie$ & 0,183 \\
\hline i) $\mathrm{Ke} \mathrm{m} \mathrm{n}$ & & & & & \\
\hline Fleischextract . & $250 \gg$ & $27 »$ & $10,80 \gg$ & $5,25 »$ & 3,50 » \\
\hline
\end{tabular}

Aus den Controlversuchen a) und b), sowie c) und d) ist ersichtlich, dass wir auf diese Weise die Phosphorfleischsäure äusserst genau bestimmen können, da eine Differenz erst in der dritten Decimale der gefundenen Procente Fleischsäure auftritt; nur wenige organische Substanzen lassen sich mit solcher Exactheit bestimmen. Da bei dem Ausfällen des Carniferrins nicht übermässig sorgfältig verfahren werden muss, indem eine eventuell erhaltene Differenz im Carniferrinniederschlag sich durch das Stickstoff verhältniss wieder ausgleicht, [vergl. Versuch a) und b)], so ist die Bestimmung der Phosphorfleischsäure nach dieser Methode auch von weniger Geübten leicht auszuführen. 
Zum Schluss sei es uns gestattet, die Methode nochmals in ihren Grundzügen zusammenzufassen.

Ca. $1 \%$, Kilo, bezw. weniger, von der Substanz, in welcher die Phosphorfleischsäure bestimmt werden soll, werden mittelst einer Maschine zerkleinert, dann genau 1 Kilo, bezw. weniger, abgewogen und zuerst zweimal mit je 2 Liter, dann einmal mit 1 Liter Wasser auf dem Wasserbade bei $50^{\circ}$ bis $60^{\circ}$ unter gutem Umrühren ungefähr 1 Stunde lang digerirt und durch ein Colirtuch gepresst. Die vereinigten Filtrate werden dann behufs Coagulation der Eiweisskörper aufgekocht und nach dem Erkalten nochmals filtrirt. Aus dem Filtrate werden die Phosphate durch eine Lösung von Calciumchlorid und Ammoniak ausgefällt. Das neutral gemachte Filtrat wird nunmehr zum Kochen erhitzt und mit einer 1 procentigen Lösung von Eisenchlorid versetzt, die man am besten aus einer Bürette hinzulässt. Durch fortwährende Tüpfelproben mit Rhodankalium sucht man einen grossen Ueberschüss von Eisenchlorid zu vermeiden, lässt, wenn Ferrireaction eintritt, ein paar Minuten kochen und hört erst dann mit dem Zusatz von Eisenchlorid auf, wenn die Ferrireaction auch nach dem Kochen noch bestehen bleibt. Hat man einen grossen Ueberschuss von Eisenchlorid zugesetzt, so stumpft man die sauere Reaction des Filtrates durch Zusatz von einigen Tropfen Ammoniak etwas ab. Die Flüssigkeit mit dem Eisenniederschlag wird nun aus den Schalen, in denen die Carniferrinfällung vorgenommen wurde, in grössere Gefässe gespült, wo sich der Niederschlag leicht absetzt und nun durch Dekantiren ausgewaschen wird, bis er nicht mehr die Salzsäurereaction gibt. Zuletzt wird der Niederschlag noch centrifugirt und mit Alkohol und Aether behandelt. In dem bei $105^{\circ}$ getrockneten Eisenniederschlag wird der Stickstoff nach Kjeldahl bestimmt und der gefundene Gesammtstickstoff auf Fleischsäure berechnet, indem man ihn mit dem Factor 6,1237 multiplicirt.

Der Eine von uns ist damit beschäftigt, topographische Bestimmungen der Phosphorfleischsäure bei verschiedenen Thieren auszuführen. 